\title{
MIGUEL ÁNGEL MARTÍNEZ ALFARO (23 DE OCtUbre De 1942 - 9 de feBReRO DE 2007)
}

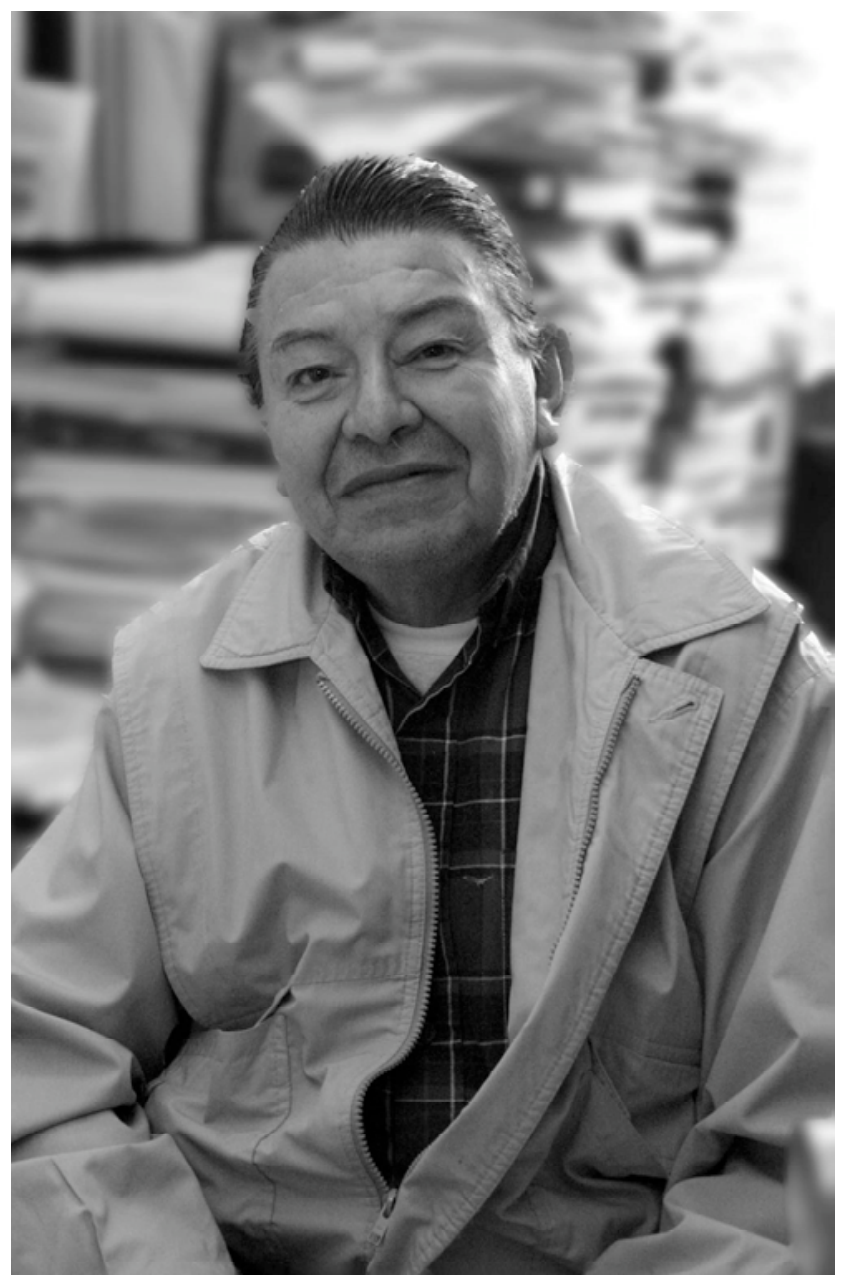

iguel Ángel Martínez Alfaro, destacado pionero en la N etnobotánica mexicana y latinoamericana, fue un hombre de gran calidad humana. Una de sus características personales más distintivas, y por la que siempre será recordado con enorme cariño, e ra su permanente disposición para compartir sus vastos conocimientos, y de ayudar, en todo lo que estaba a su alcance, a las personas que lo rodeaban. Tenía un gran sentido del humor, el que nunca olvidarán quienes lo conocieron.

Fue un destacado miembro e impulsor de la Sociedad Botánica de México desde 1965, fungiendo como Secretario de Actas de 1969 a 1972 y como Vicepresidente entre 1977 y 1978. Siempre presente en los Congresos Mexicanos de Botánica, entre sus colaboraciones para el Boletín de la Sociedad Botánica de México destaca una síntesis sobre el estado de la etnobotánica en México hacia el final del siglo pasado (Número 55, 1994). Fue un entusiasta colaborador y promotor de las excursiones botánicas que durante un tiempo la Sociedad organizaba para todo público.

Ampliamente motivado por el invaluable y rico conocimiento tradicional que poseen los pueblos indígenas de México sobre las plantas útiles, especialmente las alimenticias y las medicinales de las zonas cálido-húmedas, Miguel Ángel incursionó en la etnobotánica y la historia del uso de las plantas útiles y de la agricultura tradicional. Se ocupó de vincular a la etnobotánica con la fitogenética, en la búsqueda de recursos vegetales de valor alimenticio y agrícola, preocupándose por encontrar formas de mejorar el estado nutricional y de salud de las comunidades rurales. Junto con su compañera, la también connotada etnobotánica Abigail Aguilar Contreras, trabajó en la capacitación de promotores de la salud y en investigación histórica de la botánica médica. Impulsó la etnobotánica no sólo en México, sino también en Latinoamérica. Miguel Ángel fue, orgullosamente, uno de los alumnos más destacados de los Drs. Efraím Hernández Xolocotzi, Arturo GómezPompa y José Sarukhán, grandes botánicos mexicanos.

Lo que más disfrutaba Miguel Ángel era el poder enseñar, el ser profesor. Se trans- 
formaba cuando se le consultaba sobre algún tema; en esos casos perdía la noción del tiempo y pasaba horas y horas brindando sus conocimientos a los colegas, alumnos o al público en general. Tenía una gran capacidad para difundir los resultados científicos en un lenguaje sencillo y entendible para todos y, en particular, para los campesinos. Tenía una gran capacidad de integrarse con sus alumnos y con los campesinos con quienes colaboraba. En el trabajo de campo, los estudiantes aprendían con él, de manera directa, los conceptos botánicos, ecológicos e históricos, que además se los enriquecía con innumerables notas sobre historia y geografía de nuestro país.

Nacido en la ciudad de México el 23 de octubre de 1942, y con interés especial por los seres vivos desde su niñez, Miguel Ángel estudió biología en la Facultad de Ciencias de la Universidad Nacional Autónoma de México (UNAM) de 1961 a 1964. Al término de sus estudios, se integró a la Comisión Nacional para el Estudio de las Dioscóreas para realizar su tesis de licenciatura bajo la dirección del Dr. Arturo Gómez Pompa. Presentada en enero de 1970, su tesis, titulada Ecología humana del ejido Benito Juárez o Sabastopol, Tuxtepec, Oaxaca, es pionera y pilar de los campos de la ecología humana y la etnobotánica en México. Poco después, en julio de 1971, se convirtió en el primer egresado del ahora desaparecido Colegio Superior de Agricultura Tropical en Cárdenas, Tabasco, en donde recibió el grado de Maestro en Ciencias con la tesis Ecología humana: revisión del campo y programación del curso, realizada bajo la dirección del Ing. Efraím Hernández-Xolocotzi. Su tesis fue la base para el curso de etnobotánica que se impartió en la Escuela Nacional de Agricultura-Chapingo y en el Colegio de Posgraduados. Con un definido interés por profundizar en el entendimiento de la relación sociedad-naturaleza, de 1972 a 1973 hizo estudios de Maestría en Antropología Social en la Universidad Iberoamericana, bajo la dirección del Dr. Ángel Palerm. En 1998 ingresó al Doctorado en la Facultad de Ciencias de la UNAM, quedando inconclusa su tesis Patrones de uso de plantas en la Sierra Norte de Puebla, México.

Miguel Ángel colaboró con el Ing. Efraím Hernández-Xolocotzi en el Colegio de Postgraduados, realizando colectas de maíz y frijol en las sierras Norte de Puebla y de Huautla, en Oaxaca, y en 1976 ingresó como investigador en el ahora desaparecido Instituto Mexicano para el Estudio de las Plantas Medicinales (IMEPLAM), sentando bases para la metodología en la investigación de la etnobotánica médica en ese Instituto.

Su carrera en la UNAM como profesor e investigador se inició en febrero de 1976, cuando en la Facultad de Ciencias impartió por primera vez la cátedra de etnobotánica y la biología de campo "Uso y conocimiento diferenciado en una región cálidohúmeda de la Sierra Norte de Puebla", dejando profunda huella en el grupo de estudiantes que tuvieron la suerte de tenerlo como profesor, al igual que en todos los que posteriormente tuvieron la misma fortuna, en ésta y otras instituciones en donde impartió cátedra. Entre este primer grupo de alumnos se encuentran actuales investigadores dedicados a diferentes aspectos de la etnobotánica y la botánica, como Arturo Argueta, Cristina Mapes, Daniel Zizumbo, Guadalupe Miranda, José Arellano, Juan Manuel Rodríguez, Lourdes Villers, Martha Méndez, Patricia Colunga y Sergio Zamudio.

En julio de 1978 fue contratado como investigador de tiempo completo en el Instituto de Biología, dirigiendo exitosamente numerosos proyectos con financia- 
miento externo nacional e internacional, entre los que destacan el de "Naturaleza, Sociedad y Cultura en la Sierra Norte de Puebla", patrocinado por el Consejo Nacional de Ciencia y Tecnología (CONACYT), donde trabajó conjuntamente con el Antropólogo Elio Manferrer, de 1985 a 1987, y el de "Formación de promotores comunitarios en el Centro de Capacitación Integral para Promotores Comunitarios (CECIPRoc) en Oaxaca" con el Dr. Alberto Yzunza, de 1991 a 1994 y de 1998-1999, patrocinado por la Fundación W. Kellog. En este proyecto, que recibió el Premio Nacional de Investigación Comunitaria de la Fundación para la Investigación Comunitaria y los Laboratorios Glaxo, Miguel Ángel, junto con Abigail Aguilar, desarrollaron los curricula de las áreas de ecología y etnobotánica, donde el promotor es el principal investigador de su comunidad. Las técnicas de las caminatas botánicas y la elaboración de miniherbarios, desarrolladas por ellos en Guatemala en 1980, fueron la principal herramienta metodológica de este trabajo. El último proyecto que tuvo bajo su responsabilidad y concluyó, fue el "Inventario de frutales en huertos y cafetales de la Sierra Norte de Puebla”, realizado de 2003 a 2005 con apoyo financiero de SNICS-SAGARPA (Secretaría de Agricultura, Ganadería y Recursos Pecuarios). Vale resaltar la trascendental visión de Miguel Ángel de que el entendimiento de la relación sociedad-naturaleza requería del trabajo multidisciplinario de investigación, y su enorme habilidad para llevarlo a la práctica. Su capacidad de saber escuchar y comunicarse con los diferentes integrantes de los equipos de trabajo fue invaluable. Realizó proyectos de colaboración binacional en etnobotánica en países latinoamericanos como Colombia, con la Dra. Olga Lucía Sanabria.

Entre 1987 y 1991 Miguel Ángel fue Director del Jardín Botánico del propio Instituto de Biología. Durante su gestión dio flexibilidad y estímulo para el desarrollo de propuestas de actividades y programas educativos y apoyó las labores de difusión y educación mediante la contratación de nuevo personal para esta área. Su apoyo a la Asociación Mexicana de Jardines Botánicos se tradujo en que la Mesa Directiva de dicha asociación estuvo durante doce años a cargo del Jardín Botánico de la UNAM. Durante su período se estableció una nueva sección dedicada a la obra de la Doctora Helia Bravo-Hollis.

Publicó 5 libros, 13 capítulos de libros y 33 artículos en revistas científicas y de divulgación. Además de su ya mencionada tesis de licenciatura, un clásico de la etnobotánica mexicana, cabe destacar su libro Contribuciones iberoamericanas al mundo; botánica, medicina, agricultura (1988, Biblioteca Iberoamericana No. 27, Ediciones Anaya, S.A. Madrid) y su Catálogo de plantas útiles de la Sierra Norte de Puebla (1995, Col. Cuadernos del Instituto de Biología No. 27, Universidad Nacional Autónoma de México, México, D.F.) que comprende tres entidades federativas - Puebla, Veracruz e Hidalgo- , y que contiene gran cantidad de información histórica, etnográfica, botánica, lingüística y química de las especies citadas.

Siempre interesado en difundir su conocimiento, Miguel Ángel participó con 63 trabajos en congresos internacionales y 128 en congresos nacionales. Dictó numerosas conferencias y frecuentemente realizaba visitas guiadas y excursiones a diferentes regiones de México, a mercados, al Museo Nacional de Antropología e Historia en la ciudad de México y al Jardín Botánico de la UNAM. Participó en cuantiosos talleres y cursos que se impartieron a promotores de salud. Acudió a numerosos programas de radio donde habló sobre temas botánicos muy diversos. Recibió muchos 
agradecimientos por identificación de material botánico.

Impartió nu merosos cursos formales en dife rentes unive rsidades del país, además de la UNAM, como la Universidad Autónoma Metropolitana-Iztapalapa, la Escuela Nacional de Antropología e Historia de la Secretaría de Educación Pública, la Escuela de Antropología de la Unive rsidad Autónoma del Estado de México, la Escuela Nacional de Estudios Profesionales-Plantel Zaragoza, la Facultad de Ciencias Químicas y Biológicas de la Universidad Autónoma de Guerrero, la Escuela de Ciencias Químicas de la Universidad Autónoma Benito Juárez de Oaxaca, y las Escuelas de Agronomía y Biología de la Universidad de Guadalajara. Además, dictó cursos de etnobotánica en el Jardín Botánico de Córdoba en España. Su interés por los jóvenes lo llevó a ser participante activo del programa "Jóvenes a la Investigación" de la UNAM, recibiendo estudiantes de preparatoria, a quienes integraba en sus proyectos en la Sierra Norte de Puebla. Dirigió 34 tesis de licenciatura y 8 de maestría, varias de las cuales recibieron premios y menciones honoríficas. Dejó imborrable huella y ejemplo entre sus alumnos.

Además de su colaboración con la Sociedad Botánica de México, Miguel Ángel pertenecía a, y colaboró intensamente con, otras sociedades científicas, entre las que destacan la International Society of Ethnobiology, el Grupo Etnobotánico Latinoamericano (GELA) de la Asociación Latinoamericana de Botánica, y la Asociación Etnobiológica Mexicana. En 2006 recibió el reconocimiento a su labor y constancia en el IX Congreso Latinoamericano de Botánica. Durante el reciente IV Congreso de la Asociación Colombiana de Botánica hizo una distinción especial a Miguel Ángel "como expresión de reconocimiento y gratitud por su valiosa contribución al desarrollo de la investigación etnobotánica en Colombia y América Latina", especialmente por su calidad humana y sus enseñanzas, que enriquecieron a varias generaciones de botánicos y etnobotánicos colombianos". En el pasado VI Congreso de la Asociación Etnobiológica Mexicana, se estableció el "Premio Miguel Ángel Martínez Alfaro" que se otorgará a la mejor tesis de licenciatura en el tema.

Su colaboración con éstas y otras sociedades científicas incluyeron su participación en la organización de sus congresos. Fue parte del Comité Organizador Permanente de las "Reuniones Latinoamericanas sobre religión popular, identidad y etnociencia" y era parte del Comité Asesor y Delegado para América Latina de la International Society of Ethnobiology. De 1996 a 1999 impartió el curso intensivo de "Metodologá etnobotánica cualitativa" durante los Congresos Mexicanos de Etnobiología.

Era un enamorado y un mecenas de la cultura y la ciencia. Disfrutaba de la pintura, de la música, del cine y del teatro, sobre todo lo experimental. Fue un hombre político que militó en los partidos de la izquierda mexicana. Le encantaba viajar por el mundo al lado de su compañera Abigaíl Aguilar. Las anécdotas vividas son incontables y siempre serán recordadas.

Patricia Colunga García-Marín ${ }^{1}$, Cristina Mapes Sánchez ${ }^{2}$ y Francisco Basurto Peña ${ }^{2}$ ${ }^{1}$ Unidad de Recursos Naturales, Centro de Investigación Científica de Yucatán.

2 Jardín Botánico, Instituto de Biología, Universidad Nacional Autónoma de México. 